\title{
Insuficiencia respiratoria aguda
}

\section{Acute respiratory failure}

Carlos Bula, MD. ${ }^{(1)}$; Héctor Alejandro Zapata Oquendo, MD.(2)

\section{RESUMEN}

La insuficiencia respiratoria aguda es la disfunción del sistema respiratorio por causas pulmonares o extrapulmonares que alteran el intercambio gaseoso normal; la misma se puede clasificar en hipoxémica (PO2 <60 mm Hg), hipercápnica (PCO2 >45 mm Hg con pH < 7,3) y mixta, cuando existen criterios de las dos anteriores. Teniendo en cuenta que es una patología muy frecuente en quehacer médico, que conlleva alta mortalidad, además de grandes costos para el sistema de salud, se recomienda optimizar el tratamiento de estos pacientes a fin de disminuir las repercusiones desfavorables que, por sí misma, tiene esta entidad.

Palabras clave: insuficiencia respiratoria, hipoxémica, hipercápnica.

\section{ABSTRACT}

Acute respiratory insufficiency is the dysfunction of the respiratory system caused by pulmonary and extrapulmonary conditions, which can alter the normal gaseous exchange, this can be classified as hypoxemic $\left(\mathrm{PaO}_{2}<60 \mathrm{mmHg}\right)$, $\mathrm{Hyper}-$ capnic $\left(\mathrm{PaCO}_{2}>45 \mathrm{mmHg}\right.$ with a $\left.\mathrm{PH}<7,3\right)$ and mixed when both of the previous criteria are met. Having in mind that this condition is frequent in everyday medical experience, with a high mortality rate and great costs to our health system, we must optimize the management of these patients so that we can diminish the unfavorable repercussions of this disease.

Keywords: respiratory insufficiency, hypoxemic, hypercapnic.

Rev Colomb Neumol 2012; 23 (3): 95-102.

La insuficiencia respiratoria aguda es la disfunción del sistema respiratorio que altera el intercambio gaseoso normal y es potencialmente mortal. Cada elemento de esta definición es importante. El término aguda implica un inicio relativamente brusco (de horas o días) y un cambio sustancial de la situación basal del paciente. La disfunción de las vías respiratorias señala que el intercambio anormal de gases puede ser causado por entidades pulmonares o extrapulmonares que repercuten, directa o indirectamente, en el normal funcionamiento del sistema en mención.

La respiración, en su más amplio sentido, se refiere a la entrega de oxígeno $\left(\mathrm{O}_{2}\right)$ a los tejidos metabólicamente activos para el uso de energía y la eliminación de dióxi- do de carbono $\left(\mathrm{CO}_{2}\right)$ de estos tejidos. La insuficiencia respiratoria es un fracaso del proceso de entrega de $\mathrm{O}_{2}$ a los tejidos y/o eliminación de $\mathrm{CO}_{2}$ de los tejidos (1).

La insuficiencia respiratoria se puede dividir en hipoxémica $\left(\mathrm{PaO}_{2}<60 \mathrm{~mm} \mathrm{Hg}\right)$ e hipercápnica $\left(\mathrm{PaCO}_{2}\right.$ $>45 \mathrm{~mm} \mathrm{Hg}$ con acidemia $(\mathrm{pH}<7,3)(2)$. Así como en insuficiencia respiratoria aguda mixta, resultante de la combinación de las dos anterior es.

La insuficiencia respiratoria aguda hipercápnica, refleja bien la producción excesiva de $\mathrm{CO}_{2}$ o la eliminación inadecuada de $\mathrm{CO}_{2}$. El ejercicio, el hipertiroidismo, las quemaduras, la fiebre y la sepsis pueden incrementar la producción de $\mathrm{CO}_{2}$ por el aumento de la tasa metabólica, pero rara vez causan insuficiencia respiratoria.

\footnotetext{
(1) Neumólogo, Fundación Hospital Universitario Metropolitano, Barranquilla.

(2) Residente III Año Medicina Interna, Universidad Metropolitana, Barranquilla.
}

Correspondencia: Dr. Carlos Bula Gutierrez, correo electrónico: cjbulag@hotmail.com

Recibido: 20 de diciembre de 2011. Aceptado: 30 de diciembre de 2011 
Por el contrario, la disminución de la eliminación de $\mathrm{CO}_{2}$ refleja la falta de la unidad funcional de la ventilación, un trastorno neuromuscular.

La insuficiencia respiratoria aguda tipo II se produce sólo cuando el paciente tiene acidosis asociada, lo cual implica que el cambio en el $\mathrm{CO}_{2}$ fue demasiado rápido o extremo para la compensación renal. De otra parte, el pH indica si la hipercapnia es aguda o crónica (3).

La insuficiencia respiratoria aguda hipoxémica, convencionalmente definida como una presión arterial de oxígeno $\left(\mathrm{PaO}_{2}\right)$ menor a $60 \mathrm{~mm} \mathrm{Hg}$. crea controversia porque hace caso omiso de la fracción inspirada de oxígeno $\left(\mathrm{FiO}_{2}\right)$, razón por la cual algunos prefieren la relación $\mathrm{PaO}_{2} / \mathrm{FiO}_{2}$ menor de 300 para considerar insuficiencia respiratoria aguda (4).

La insuficiencia respiratoria aguda tiene una presentación clínica variada, que puede ser originada por innumerable cantidad de patologías pulmonares y extrapulmonares, razón por la que su manejo y pronóstico son distintos.

La lesión pulmonar aguda (LPA) (5) y el síndrome de dificultad respiratoria aguda (SDRA) como expresión de insuficiencia respiratoria aguda hipoxémica, son trastornos devastadores que afectan hasta a 200.000 pacientes cada año en los Estados Unidos (6) (Tabla 1).

\section{INSUFICIENCIA RESPIRATORIA AGUDA}

Remitirse a Tabla 1.

\section{SISTEMA DE ÓRGANOS IMPLICADOS}

1. Respiratorio (pulmones y tórax)

- Obstrucción al flujo aéreo

- EPOC

- Asma
- Parénquima pulmonar

- Neumonía

- Lesión pulmonar aguda/SDRA

- Exacerbación de enfermedad del colágeno (síndrome de Goodpasture o LES).

2. Sistema nervioso central

- Depresión respiratoria

- Sedantes, ansiolíticos, opiáceos, alcohol

- Tronco encefálico y médula espinal

- Tumores, traumatismos, accidentes vasculares

3. Neuromusculares

- $\quad$ Síndrome de Guillain-Barré

- Miastenia gravis

4. Cardiovascular

- Edema pulmonar

- Tromboembolismo pulmonar

5. Insuficiencia renal/endocrino

- Sobrecarga de volumen

- Anomalías metabólicas

La hipoxemia puede ser resultado de varias alteraciones como es la disminución del FIO2, hipo ventilación, deterioro de la difusión, trastornos en la ventilación-perfusión o por shunt, los cuales van en detrimento de la fisiología de la respiración, provocando trastornos en los distintos componentes que intervienen en el intercambio gaseoso (Tabla 2).

\section{DIAGNÓSTICO DIFERENCIAL DE HIPOXEMIA}

Remitirse a la Tabla 2. Mecanismos fisiopatológicos de la insuficiencia respiratoria aguda.

Tabla 1. Causas de insuficiencia respiratoria aguda.

\begin{tabular}{|ll|}
\hline Insuficiencia respiratoria aguda hipoxémica & Insuficiencia respiratoria aguda hipercápnica \\
\hline Lesión pulmonar aguda/SDRA & Enfermedad pulmonar (EPOC, asma, EPID) \\
Neumonía & Drogas (opiáceos, ansiolíticos...) \\
Tromboembolismo pulmonar & Neuromusculares (síndrome de Guillain-Barré \\
& Miastenia gravis, lesión del nervio frénico) \\
Atelectasia & Metabólicas (hipofosfatemias, hipomagnesemia) \\
Edema pulmonar & Músculo-esqueléticas \\
Contusión pulmonar & Síndrome de obesidad-hipoventilación \\
Enfermedad del colágeno (LES, síndrome de Goodpasteur) & Debilidad muscular post-ventilación mecánica prolongada
\end{tabular}




\section{Insuficiencia respiratoria aguda hipoxémica}

El mecanismo más común es la alteración en la ventilación-perfusión, que se caracteriza por un ampliado gradiente $\mathrm{A}-\mathrm{a} \mathrm{O}_{2}$, un aumento dramático en la $\mathrm{PaO}_{2}$ en respuesta a $\mathrm{O}_{2}$ suplementario, y una $\mathrm{PaCO}_{2}$ variable. Cuando las áreas de baja $V / Q$ predominan (por ejemplo, reducción de la ventilación con perfusión normal), la $\mathrm{PaCO}_{2}$ puede ser baja ya que el paciente hiperventila en un esfuerzo (sólo parcialmente efectivo) para aumentar la $\mathrm{PaO}_{2}$. Cuando las zonas de V/Q alta predominan, la ventilación se pierde y la hipercapnia se hace presente. Las áreas de los pulmones que son perfundidas pero no ventiladas caracterizan el shunt. La consiguiente caída en la $\mathrm{PaO}_{2}$ depende del porcentaje del gasto cardíaco que circula a través del shunt y el contenido de $\mathrm{O}_{2}$ de la sangre. $\mathrm{El} \mathrm{O}_{2}$ suplementario tiene un efecto mínimo o pequeño en la $\mathrm{PaO}_{2}$ porque en la sangre desviada no esta en contacto con los capilares para producir el intercambio gaseoso (Tabla 3 , figura 1). Por lo tanto, el tratamiento está dirigido a disminuir el shunt mediante la mejoría de la ventilación en el área afectada o la reducción de la perfusión en la misma. Cuando el shunt es producto de un proceso unilateral (neumonía, atelectasia, entre otras), ubicar el pulmón sano hacia abajo, disminuye la perfusión del shunt y mejora la oxigenación.

\section{Insuficiencia respiratoria aguda hipercápnica}

Se define como una $\mathrm{PaCO}_{2}$ superior a $45 \mathrm{~mm} \mathrm{Hg}$ asociada a acidemia. La ecuación utilizada para determinar la $\mathrm{PaCO}_{2}$ da una idea de los tres mecanismos básicos subyacentes de hipercapnia:

$$
\mathrm{PaO}_{2}=\mathrm{k}\left(\mathrm{VCO}_{2}\right) / \mathrm{VE}-1(\mathrm{VD} / \mathrm{VT})
$$

Tabla 2. Diagnóstico diferencial de hipoxemia.

\begin{tabular}{|c|c|c|c|}
\hline Causas de hipoxemia & Mecanismos de hipoxemia & Enfermedades asociadas & Comentarios \\
\hline Disminución de $\mathrm{FiO}_{2}$ & $\begin{array}{l}\text { Presión atmosférica a gran altura } \\
\text { o en aviones } \\
\text { Presión alveolar de } \mathrm{O}_{2} \text {. }\end{array}$ & & $\begin{array}{l}\text { Pocas veces clínicamente signifi- } \\
\text { cativo }\end{array}$ \\
\hline Hipoventilación & $\begin{array}{l}\text { La concentración alveolar de } \mathrm{CO}_{2} \\
\text { disminuye la concentración de } \\
\mathrm{O}_{2} \text { alveolar }\end{array}$ & & $\begin{array}{l}\text { Corregir con una pequeña cantidad } \\
\text { de } \mathrm{O}_{2} \text { suplementario } \\
\text { Desaturación con un mínimo esfuer- } \\
\text { zo DLCO }\end{array}$ \\
\hline Deterioro de la difusión & $\begin{array}{l}\text { Aumento del tiempo para que el } \\
\mathrm{O}_{2} \text { pase a través de la membrana } \\
\text { alvéolo-capilar o la pérdida de la } \\
\text { superficie total alvéolo-capilar } \\
\text { disminuya la entrega de } \mathrm{O}_{2} \text { a la } \\
\text { hemoglobina }\end{array}$ & Enfermedad pulmonar intersticial & $\begin{array}{l}\text { Incremento del gradiente A-a. } \\
\text { Normal o bajo } \mathrm{PCO}_{2} \\
\mathrm{PaO}_{2} \text { con oxígeno } \mathrm{O}_{2} \text { suplementario }\end{array}$ \\
\hline $\begin{array}{l}\text { Trastorno ventilación- } \\
\text { perfusión }\end{array}$ & $\begin{array}{l}\text { La alteración de la relación } \\
\text { ventilación-perfusión reduce la } \\
\text { eficiencia del intercambio gaseo- } \\
\text { so y disminuye el aporte de } \mathrm{O}_{2} \text { a } \\
\text { la hemoglobina }\end{array}$ & $\begin{array}{l}\text { Enfermedades de la vía aérea } \\
\text { (asma, EPOC, neumonía, bron- } \\
\text { quitis) }\end{array}$ & \\
\hline Shunt & $\begin{array}{l}\text { La sangre venosa mixta omite } \\
\text { la función pulmonar y baja la } \\
\text { presión de } \mathrm{O}_{2} \text { sistémico }\end{array}$ & $\begin{array}{l}\text { Enfermedad pulmonar intersticial. } \\
\text { Enfermedad vascular pulmonar } \\
\text { (embolia, hipertensión pulmonar) } \\
\text { Enfermedad alveolar (SDRA, } \\
\text { atelectasias, edema pulmonar, } \\
\text { neumonía) } \\
\text { Enfermedad vascular (malfor- } \\
\text { mación arteriovenosa, síndrome } \\
\text { hepatorrenal) } \\
\text { Intracardiaca (defectos septales) }\end{array}$ & $\begin{array}{l}\text { Incremento del gradiente } \mathrm{A}-\mathrm{a} \\
\text { Normal o leve elevación de } \mathrm{PCO}_{2} \\
\mathrm{PO}_{2} \text { no se corrige con } \mathrm{O}_{2} \text { suple- } \\
\text { mentario }\end{array}$ \\
\hline
\end{tabular}

Gradiente A-a (gradiente alveolar-arterial). DLCO: capacidad de difusión del monóxido de carbono; FiO ${ }_{2}$ fracción inspirada de oxígeno; $\mathrm{PaO}_{2}$ : Presión arterial de oxígeno; $\mathrm{PCO}_{2}$ : presión parcial de dióxido de carbono; $\mathrm{PO}_{2}$ : presión parcial de oxígeno. 
Donde k es una constante, VE es la ventilación total por minuto (frecuencia respiratoria por el volumen corriente) y VD/VT es el espacio muerto. Por lo tanto, la hipercapnia puede ser resultado de una mayor producción de $\mathrm{CO}_{2}\left(\mathrm{VCO}_{2}\right)$, aumento del espacio muerto fisiológico (VD/NT), y disminución de la ventilación por minuto. El aumento de la $\mathrm{VCO}_{2}$ solo es por lo general insuficiente para provocar hipercapnia porque el sistema respiratorio responde incrementando la ventilación por minuto para mantener la $\mathrm{PaCO}_{2}$ normal. A la inversa, con anomalías de los músculos respiratorios, una unidad respiratoria o con aumento del espacio muerto (y disminución en la reserva), la respuesta respiratoria para aumentar $\mathrm{VCO}_{2}$ puede ser insuficiente y dar como resultado hipercapnia.

\section{CLÍNICA}

El cuadro clínico de la insuficiencia respiratoria aguda hipoxémica puede dividirse en:

1. Síntomas iniciales inespecíficos: tos, expectoración, disnea.

\section{Examen clínico:}

a. Signos de dificultad respiratoria o evidencia de aumento del trabajo respiratorio: taquipnea (FR $>30 \mathrm{x}$ min) y disnea (percepción de falta de aire). Esto puede ser variable entre un observador y otro, hasta en $35 \%$. Similar limitante existe entre hipoxemia y frecuencia respiratoria; así, hasta $44 \%$ de los pacientes con saturación arterial menor a $90 \%$ presentan frecuencia respiratoria dentro de límites normales. Adicionalmente, puede haber aleteo nasal, uso de músculos accesorios y retracciones intercostales.

b. Cianosis con suplencia de oxígeno: la presencia de cianosis distal en pacientes que reciben suplemento de oxígeno superior al $40 \%$ es un buen predictor de insuficiencia respiratoria aguda.

c. Signos de fatiga muscular: en los músculos respiratorios el trabajo excesivo causa fatiga; sin embargo el concepto de esta última es difícil de identificar en la práctica clínica.

Una de las pocas manifestaciones clínicas de fatiga muscular son los movimientos paradójicos abdominales o la respiración paradójica.

d. Otros: mientras que la hipertensión y la taquicardia son signos de respuesta inespecífica, la presencia de diaforesis, hipotensión, bradicardia y alteración de conciencia son signos de insuficiencia respiratoria aguda y se asocian con mal pronóstico.
Las entidades particulares tienen hallazgos que pueden orientar a su diagnóstico. Así, los signos clásicos de neumonía como ruidos respiratorios disminuidos, estertores, roncus, broncofonía, pectoriloquia y matidez pueden tener una alta variabilidad; no obstante, su presencia asociada con los síntomas y signos de una buena historia clínica, facilitarán el enfoque diagnóstico y terapéutico a seguir. El edema, la ortopnea, los estertores y el aumento en el pulso venoso sugieren edema pulmonar cardiogénico. Enfermedades como la EPOC y el asma tienen signos clínicos similares, pero una historia clínica completa permitirá esclarecer el dictamen. En el diagnóstico diferencial deben incluirse patologías como: neumotórax espontáneo, atelectasias y tromboembolismo pulmonar.

3. En el caso de la insuficiencia respiratoria aguda hipercápnica el cuadro clínico dependerá de la enfermedad de base. A esto se asociarán signos y síntomas ocasionados por la retención de $\mathrm{CO}_{2}$ tales como cefalea, vasodilatación facial y conjuntivas hiperémicas. En cuadros severos puede haber asterixis, somnolencia y coma (7-19).

\section{DIAGNÓSTICO}

\section{Evaluación de la oxigenación}

El monitoreo de esta variable se puede hacer en forma discontinua con determinación de gases arteriales, o continua mediante oximetría de pulso. Como valores limítrofes a obtener con suplencia de oxígeno, se aceptan: saturación arterial de oxígeno $\left(\mathrm{SaO}_{2}\right) \mathrm{ma}$ yor a $90 \%-92 \%, \mathrm{PaO}_{2}$ mayor a $60 \mathrm{~mm} \mathrm{Hg}$ y $\mathrm{PaO}_{2} / \mathrm{FiO}_{2}$ mayor a $200 \mathrm{~mm} \mathrm{Hg}$.

\section{Evaluación de la ventilación}

El monitoreo de la ventilación se realiza mediante la toma de gases arteriales, procedimiento invasivo e intermitente, o por el registro continuo del $\mathrm{CO}_{2}$ espirado medido por un capnógrafo. Anteriormente, un nivel aislado de $\mathrm{PaCO}_{2}$ era un dato suficiente para diagnosticar falla ventilatoria; sin embargo, dada la creciente cantidad de pacientes retenedores crónicos de $\mathrm{CO}_{2}$ (EPOC), se considera necesaria la asociación de hipercapnia con un pH menor a 7,30; esto último definiría la agudización.

\section{Radiografía de tórax}

Ésta se ha evaluado en múltiples estudios que incluyen pacientes con insuficiencia respiratoria aguda, y si bien puede haber una amplia variabilidad dependiente del observador, es clara su utilidad: 
1. Diagnóstico primario: la radiografía de tórax permite el diagnóstico de patologías como edema pulmonar cardiogénico, neumotórax, atelectasia y derrame pleural. En pacientes con politraumatismo es fundamental en el diagnóstico de fracturas costales o de lesiones de otras estructuras de la pared torácica, así como hemotórax, neumotórax, contusión pulmonar, entre otras.

2. La radiografía de tórax normal en pacientes con insuficiencia respiratoria aguda a descartar diagnósticos diferenciales ya mencionados. Igualmente debe hacer pensar en las pocas patologías que ocasionan insuficiencia respiratoria aguda con radiografía de tórax normal, entre éstas el asma y el tromboembolismo pulmonar.

\section{TRATAMIENTO}

El tratamiento de la insuficiencia respiratoria aguda consiste en controlar la causa de la insuficiencia en combinación con un soporte en oxigenación y ventilación.

El abordaje inicial del paciente permitirá:

- Corregir los gases arteriales, hipoxemia y/o hipercapnia sin que esto genere riesgos de baro o volutrauma.

- Determinar el requerimiento de ventilación no invasiva.

- Establecer la necesidad de intubación e iniciar ventilación invasiva.
- Fijar la etiología mediante estudios paraclínicos dirigidos de acuerdo con los hallazgos en interrogatorio y examen.

- Formular un plan diagnóstico cuando la etiología no sea evidente.

\section{Insuficiencia respiratoria hipercápnica}

El manejo inicial consiste en permeabilizar la vía aérea por terapia respiratoria como se describirá más adelante. De no observarse mejoría y ante la imposibilidad de proteger la vía aérea por el paciente, se procederá a la intubación orotraqueal y/o la traqueostomía. La hipercapnia es sinónimo de hipoventilación alveolar; es así como los soportes ventilatorios restauran esta ventilación a la espera de corregir la patología o causa de descompensación. Es necesario aclarar que en pacientes con insuficiencia respiratoria aguda hipercápnica, EPOC en particular, la literatura ha demostrado la utilidad de la ventilación mecánica no invasiva.

La hipoxemia se presenta también en falla respiratoria hipercápnica especialmente en pacientes con enfermedad pulmonar, de ahí que sea necesario el aporte suplementario de oxígeno; no obstante, algunos casos son particularmente insensibles a la hipercapnia y requieren el estímulo de la hipoxemia para activar el centro ventilatorio. Si se administra una suplencia de oxígeno que corrija completamente la hipoxemia, se podría inhibir el centro respiratorio e incrementar la hipoventilación.

Tabla 3. Mecanismos fisiopatológicos de la hipoxemia.

\begin{tabular}{|c|c|c|c|c|}
\hline Mecanismo & Gradiente $\mathrm{A}-\mathrm{a} \mathrm{O}_{2}$ & $\mathrm{PCO}_{2}$ & Respuesta $\mathrm{O}_{2} 100 \%$ & Causas \\
\hline $\begin{array}{l}\text { Alteración de la di- } \\
\text { fusión }\end{array}$ & Aumentado & $\begin{array}{l}\text { Aumentado } \\
\text { Normal }\end{array}$ & Buena & $\begin{array}{l}\text { EPID } \\
\text { Sobredosis de narcóti- }\end{array}$ \\
\hline Hipo ventilación & Normal & Aumentado & Buena & $\begin{array}{l}\text { cos, síndrome de hipo- } \\
\text { ventilación alveolar }\end{array}$ \\
\hline $\begin{array}{l}\text { Disminución de la } \\
\mathrm{FiO}_{2}\end{array}$ & Normal & Usualmente disminuido & Buena & Grandes altitudes \\
\hline $\begin{array}{l}\text { Disminución de la sa- } \\
\text { turación venosa mixta }\end{array}$ & Aumentado & Usualmente disminuido & Leve & ALI, shock, EP \\
\hline Shunt & Aumentado & Usualmente disminuido & No o leve & $\begin{array}{l}\text { ALI, atelectasia, } \\
\text { EP }\end{array}$ \\
\hline Desequilibrio V/Q & Aumentado & $\begin{array}{l}\text { Aumentado } \\
\text { Normal o disminuido }\end{array}$ & Buena & $\begin{array}{l}\text { Exacerbación de } \\
\text { EPOC, asma, EP }\end{array}$ \\
\hline
\end{tabular}

EPID: enfermedad pulmonar intersticial difusa, ALI: lesión pulmonar aguda. EP: embolismo pulmonar. 


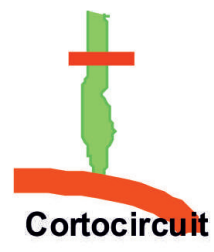

$\circ$

0/Q

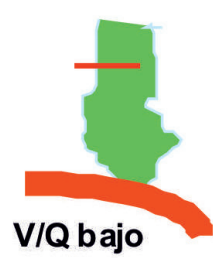

VIQ

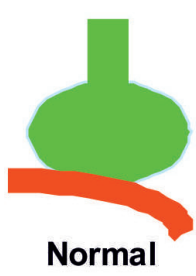

V/Q

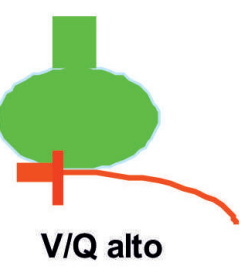

VIQ

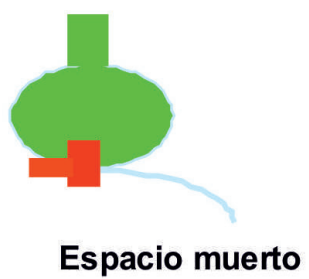

V/0

Figura 1. Mecanismos de insuficiencia respiratoria aguda hipoxémica.

\section{Insuficiencia respiratoria hipoxémica}

La suplencia o el soporte con oxígeno es la terapia más importante. Existen medidas alternativas según la causa o enfermedad que esté generando la insuficiencia. Entre ellas se encuentran el uso de PEEP en SDRA, antibióticos en neumonía y broncodilatadores en procesos bronco-obstructivos. En algunos casos la hipoxemia puede acompañarse de hipercapnia, en especial cuando el trabajo respiratorio exagerado lleva a fatiga muscular.

\section{Vía aérea}

La obstrucción de la vía aérea superior (OVAS) origina falla respiratoria en algunos casos y obliga a la corrección de la obstrucción y/o la instauración de una vía aérea artificial. Se considera en todos los pacientes con dificultad respiratoria de causa no clara, en especial si se asocia a trauma de cara o cuello, sospecha de malignidad en laringe o tráquea y enfermedad neurológica o si el cuadro se presenta en forma súbita en asociación con estridor laríngeo. Si existe antecedente de intubación previa, se plantea la posibilidad de una estenosis subglótica.

\section{Oxígeno}

Es necesario en todos los pacientes con insuficiencia respiratoria aguda y puede administrarse de dos formas:

- Sistemas de bajo flujo: incluyen las cánulas nasales y las máscaras simples. Son más cómodos para el paciente pero su limitante consiste en que el flujo de oxígeno suplementario es constante y la fracción inspirada de oxígeno varía inversamente con la ventilación minuto del paciente (VE). El flujo de oxígeno para una cánula nasal puede ser de 0,5 a $4 \mathrm{~L} / \mathrm{min}$ mientras que para una máscara es de 4-8 $\mathrm{L} / \mathrm{min}$ con la intención de barrer el $\mathrm{CO}_{2}$ existente en ésta.
- Sistemas de alto flujo: incluyen las máscaras tipo Ventury y las de reserva. Los dispositivos tipo Ventury dirigen el oxígeno a través de un pequeño tubo que incrementa la velocidad del flujo, generando mayor presión negativa y por lo tanto mayor volumen de aire en el circuito respiratorio; éstas permiten además variar la $\mathrm{FiO}_{2}$ de 0,24 hasta 0,5 , requiriendo la administración de un flujo entre $10-15 \mathrm{~mL} / \mathrm{min}$. Si bien pueden ser incómodas para el paciente tienen la ventaja de garantizar una $\mathrm{FiO}_{2}$ constante no dependiente de VE.

\section{Toxicidad del oxígeno}

Debe recordarse su posible toxicidad y efectos adversos al usar dosis altas, especialmente en pacientes con insuficiencia respiratoria aguda hipercápnica (depresión respiratoria con mayor aumento de $\mathrm{PaCO}_{2}$ ). En general, se recomienda no usar fracciones inspiradas mayores de $60 \%$ y por tiempos prolongados, mayores de seis horas.

\section{Uso de medicamentos}

Fármacos como: broncodilatadores, beta-agonistas, anticolinérgicos, esteroides, xantinas, sulfato de magnesio, sedantes y relajantes musculares, hacen parte del arsenal terapéutico para el tratamiento de estos pacientes.

\section{VENTILACIÓN MECÁNICA NO INVASIVA EN INSUFICIENCIA RESPIRATORIA AGUDA POR EDEMA AGUDO DE PULMÓN}

Es una revisión sistemática y en un meta-análisis (20), se demuestra la eficacia de la ventilación mecánica no invasiva (VMNI) para reducir la intubación y la tasa de mortalidad de los pacientes con edema agudo de pulmón. En una revisión sistemática anterior, publicada en 1998, la CPAP (una modalidad de ventilación no invasiva que proporciona una presión positiva a la vía aérea facilitando la mecánica respiratoria del paciente; 
es decir, aumenta la ventilación alveolar sin requerir la creación de una vía artificial), se asoció con una disminución en la necesidad de intubación (diferencia de riesgo $-26 \%$ ) y una tendencia a disminuir la mortalidad (21) , pero no había pruebas suficientes sobre la eficacia de VPSNI, ya fuera en comparación con el tratamiento estándar o CPAP, puesto que no había ensayos al azar en ese momento.

No obstante, en los últimos años se han desarrollado múltiples estudios donde sí se evalúan CPAP o VPSNI en pacientes con edema agudo de pulmón. Probablemente como consecuencia del aumento de tamaño de la muestra, este meta-análisis reforzó, de forma evidente, el papel de la CPAP en comparación con la terapia convencional, mostrando una dramática reducción en la necesidad de intubación (reducción del riesgo del $60 \%$ ) y una disminución de la mortalidad (47\%), estadísticamente significativa. De manera paralela a estos resultados, VPSNI demostró una reducción similar en la necesidad de intubación (52\%) y una tendencia a disminuir la mortalidad en comparación con la terapia convencional. En el cotejo de las modalidades de VNI, la VPSNI tiene ventaja sobre CPAP por cuanto ayuda a los músculos de las vías respiratorias durante la inspiración, lo que se traduce en un más rápido alivio de la disnea y la extenuación (22). Empero, estos beneficios fisiológicos no se tradujeron en los resultados primarios de ese meta-análisis, que no encontró diferencias entre CPAP y VPSNI en términos de intubación o mortalidad (23).

\section{VENTILACIÓN MECÁNICA NO INVASIVA EN ENFERMEDAD PULMONAR OBSTRUCTIVA CRÓNICA}

En múltiples estudios se sustenta que la VMNI es la mejor opción para el tratamiento de la insuficiencia respiratoria aguda hipercápnica por enfermedad pulmonar obstructiva crónica. Así, por ejemplo, en un estudio realizado en la sala general de un servicio de Neumología, se mostró como la VMNI es una alternativa terapéutica útil en más del $80 \%$ de pacientes con insuficiencia respiratoria aguda hipercápnica asociada a la reagudización de la EPOC (24).

Durante los últimos años la VNI ha adquirido progresivamente mayor protagonismo en el tratamiento del fracaso ventilatorio agudo de los pacientes con EPOC, debido a que reduce la mortalidad y las complicaciones asociadas con la intubación orotraqueal (25), además de ser altamente costo-efectiva (26). En un estudio multicéntrico realizado durante dos años en catorce hospitales, Plant y colaboradores objetivaron que la VNI reducía la frecuencia de las intubaciones orotraqueales y la mortalidad asociada (27).

\section{VENTILACIÓN MECÁNICA INVASIVA}

La VMI se debe iniciar ante la presencia de criterios definidos ampliamente en la literatura médica o según juicio clínico. En esta estrategia, la oxigenación se manipula con la fracción inspirada de oxígeno y la PEEP mientras que la ventilación se manipula con la frecuencia respiratoria y el volumen corriente.

\section{Criterios de VMI:}

1. Dificultad respiratoria y deterioro neurológico.

2. Dificultad respiratoria y deterioro cardiovascular.

3. Tórax paradójico.

4. FR> 35 ó < 6 rpm.

5. $\mathrm{PaCO}_{2}>50$ y $\mathrm{pH}<7.30$.

6. $\mathrm{PaO}_{2} / \mathrm{FIO}_{2}<200$.

\section{CONCLUSIÓN}

La insuficiencia respiratoria aguda es una patología frecuente, compleja y multifactorial. Clínicamente, en sus estadios iniciales se observan manifestaciones inespecíficas como disnea, tos y expectoración que rápidamente progresan a aumento del trabajo respiratorio, signos de fatiga muscular e inestabilidad hemodinámica, además de síntomas y signos asociados a retención de CO2 en la insuficiencia respiratoria aguda hipercápnica. Es imperativo, entonces, evaluar la oxigenación por medio de la $\mathrm{PaO} 2 / \mathrm{FiO} 2$ preferentemente, así como la ventilación por medio de $\mathrm{PaCO} 2$ o el registro $\mathrm{CO} 2$ espirado medido por un capnógrafo; esta última opción es poco habitual en nuestro medio. El tratamiento de estos pacientes es dinámico e interdisciplinario. De igual forma, se requiere tener presentes los criterios de ventilación mecánica para ofrecerla oportunamente o según el juicio clínico, ya que es preciso evitar al máximo la hipoperfusión tisular que rápidamente conduce al fallo de otros órganos y al aumento de la morbimortalidad, la cual, por sí sola, es bastante elevada.

\section{BIBLIOGRAFÍA}

1. Leonard D. Hudson. Arthur S. Slutsky. Acute respiratory failure. Goldman:Cecil Medicine, 24ed 2011.104: 629-638.

2. Franco D'Alessio, MD et al, Acute respiratory failure; Piccini \& Nilsson. 2nd ed. 2006.18:218-227

3. Hudson LD, Slutsky AS. Acute respiratory failure. Goldman: Cecil Medicine, 24 ed. 2011; 104: 629-638.

4. Epstein SK. Bope and Kellerman: Conn's Current Therapy 2012 , 1st. ed. 2011; 6: 322-327.

5. Salim A, Martin M. High-frequency percussive ventilation. Crit Care Med 2005; 33: S241-5. 
6. Rubenfeld GD, Caldwell E, Peabody E, Weaver J, Martin DP, Neff M, Stern EJ, Hudson LD. Incidence and outcomes of acute lung injury. N Engl J Med 2005; 353: 1685-93.

7. Dobbs LG, González R, Matthay MA, et al. Highly water permeable type I alveolar epithelial cells confer high water permeability between the airspace and vasculature in rat lung. Proc Natl Acad Sci U S A 1998; 95: 2991-2996.

8. Ridge KM, Rutschman $\mathrm{DH}$, Factor $\mathrm{P}$, et al. Differential expression of Na-K-ATPase isoforms in rat alveolar epithelial cells. Am J Physiol Lung Cell Mol Physiol 1997; 273: L246-L255.

9. Borok Z, Liebler JM, Lubman RL, et al. Sodium transport proteins are expressed by rat alveolar epithelial type I cells. Am J Physiol Lung Cell Mol Physiol 2002; 282: L599-L608.

10. Johnson MD, Widdicombe JH, Allen L, et al. Alveolar epithelial type I cells contain transport proteins and transport sodium, supporting an active role for type I cells in regulation of lung liquid homeostasis. Proc Natl Acad Sci U S A 2002; 99: 1966-1971.

11. Folkesson HG, Matthay MA. Alveolar epithelial ion and fluid transport. Recent progress. Am J Respir Cell Mol Biol 2006; 35: 10-19.

12. Taylor AE, Guyton AC, Bishop VS. Permeability of the alveolar epithelium to solutes. Circ Res 1965; 16: 353-362.

13. Berthiaume Y, Broaddus VC, Gropper MA, et al. Alveolar liquid and protein clearance from normal dog lungs. J Appl Physiol 1988; 65: 585-593.

14. Staub NC: Pulmonary edema. Physiol Rev 1974; 54: 678-811.

15. Basset G, Crone C, Saumon G. Fluid absorption by rat lung in situ: Pathways for sodium entry in the luminal membrane of alveolar epithelium. J Physiol (Lond) 1987; 384: 325-345.

16. Matthay MA, Folkesson HG, Verkman AS. Salt and water transport across alveolar and distal airway epithelium in the adult lung. Am J Physiol Lung Cell Mol Physiol 1996; 270: L487-L503.
17. Sakuma T, Okaniwa G, Nakada T, et al. Alveolar fluid clearance in the resected human lung. Am J Respir Crit Care Med 1994; 150: $305-310$.

18. Weibel ER. Lung morphometry and models in respiratory physiology. In: Chang HK, Paiva M, ed. Respiratory Physiology: An Analytical Approach, New York: Marcel Dekker; 1989. p. 1-56.

19. Schneeberger-Keeley EE, Karnovsky MJ. The ultrastructural basis of alveolar-capillary membrane permeability to peroxidase used as a tracer. J Cell Biol 1968; 37: 781-793.

20. Masip J, Marta R, et al. Systematic review and meta-analysis. JAMA 2005; 294 (24)

21. Pang D, Keenan SP, CookDJ, Sibbald WJ. The effect of positive pressure airway support on mortality and need for intubation in cardiogenic pulmonary edema: a systematic review. Chest 1998; 114: 1185-1192.

22. Winsock M. Noninvasive ventilation in acute cardiogenic pulmonary edema: better than continuous positive airway pressure? Intensive Care Med 1999; 25: 1-2.

23. González Barca FJ, Zamarrón Sanz LC, Salgueiro Rodríguez M, Rodríguez Suárez JR. Ventilación no invasiva en pacientes con enfermedad pulmonar obstructiva crónica e insuficiencia respiratoria aguda hipercápnica en una sala de hospitalización convencional An Med Int 2004; 21 (8): 373-377.

24. Fernández Guerra J, López-Campos Bodineau JL, et al. Metanálisis de la eficacia de la ventilación no invasiva en la exacerbación aguda de la enfermedad pulmonar obstructiva crónica. Med Clin (Barc) 2003; 120: 281-286

25. Plant PK, Owen JL, Parrott S, Elliott MW. Cost effectiveness of ward based non-invasive ventilation for acute exacerbations of chronic obstructive pulmonary disease: economic analysis of randomized controlled trial. BMJ 2003; 326: 1-5.

26. Plant PK, Owen JL, Elliott MW. Early use of non-invasive ventilation for acute exacerbations of chronic obstructive pulmonary disease on general respiratory wards: a multicentre randomized controlled trial. Lancet 2000; 355: 1931-1935.

27. Dueñas $\mathrm{C}$, et al. Hacer el bien, bien hecho en UCI. Acta Colombiana de Cuidado Intensivo 2009; 9 (Supl. 2). 